\title{
ANDRONOV-HOPF'S BIFURCATION IN A DYNAMIC MODEL OF CELL POPULATION
}

\author{
Ju.G. Nekhozhina, V.A. Sobolev \\ Samara National Research University, Samara, Russia
}

\begin{abstract}
The mathematical model of the differential cascade with a symmetrical division of stem cells is considered in the paper. The using of the RouthHurwitz's criterion and the Andronov-Hopf theorem [1] allows to find the conditions of bifurcation cycle in the model under consideration, and the phenomenon of "soft loss of stability" was stated.
\end{abstract}

Keywords: bifurcation of the cycle, stability, dynamic model, cell population

Citation: Nekhozhina JuG, Sobolev VA. Andronov-Hopf's bifurcation in a dynamic model of cell population. CEUR Workshop Proceedings, 2016; 1638 636-641. DOI: 10.18287/1613-0073-2016-1638-636-641

\section{Introduction}

Symmetric division of stem cells is a key way to increase the number of cells in development and regeneration of tissue after injury. However, this can lead to uncontrolled growth of malignant tumors. The modeling of the emergence and growth of malignant tumors is certainly one of the main directions of mathematical modeling in biology.

In order to examine the role of symmetric cell division a mathematical model of cell population was considered. The paper examines various dimensions of the system that describes this model. The aim of this work is to find the minimum value of the number of equations in the system at which stability is lost. Analytically the conducted analysis has allowed to establish the loss of stability, when it is a low-dimensional problem, and to confirm this fact by numerical experiments.

\section{Model description}

Dynamic model of cell population can be represented by the following system of ordinary differential equations [2]: 


$$
\left\{\begin{array}{l}
\dot{x}_{0}=p x_{0}-d_{0} x_{0}-\lambda_{0} x_{0} x_{n+1}, \\
\dot{x}_{i}=2 d_{i-1} x_{i-1}-\lambda_{i} x_{i}-d_{i} x_{i}, i=1, \ldots, n-1, \\
\dot{x}_{n}=2 d_{n-1} x_{n-1}-\lambda_{n} x_{n}, \\
\dot{x}_{n+1}=s x_{n}-\lambda_{n+1} x_{n+1}-\lambda_{0} x_{0} x_{n+1} .
\end{array}\right.
$$

In this system: $x_{0}$ represents the number of stem cells, $x_{i}$, with $i=1, \ldots, \mathrm{n}-1$, the number of cells in the $i$ compartment at the metastable state, and $x_{n}$ is the number of mature cells, $x_{n+1}$ represents the amount of a cytokine which regulates the size of the stem cells compartment through a negative-feedback, $p$ represents growth rate of stem cells, $d_{i}$, with $i=1, \ldots, \mathrm{n}$, is the number of divided cells per day, $\lambda_{i}$, with $i=1, \ldots, \mathrm{n}$, represents the speed of death of the relevant cells, $s$ is a positive parameter.

The problem consists in finding the minimum value of $n$ at which the system`s stability is lost. When $n=0$ the steady state with positive coordinates is asymptotically stable. So, let start with $n=1$.

\section{The study of the stability of the system when $n=1$}

In this case, the system has three equations. This system looks as follows:

$$
\left\{\begin{array}{l}
\dot{x}_{0}=p x_{0}-d_{0} x_{0}-\lambda_{0} x_{0} x_{2}, \\
\dot{x}_{1}=2 d_{0} x_{0}-\lambda_{1} x_{1}, \\
\dot{x}_{2}=s x_{1}-\lambda_{2} x_{2}-\lambda_{0} x_{0} x_{2} .
\end{array}\right.
$$

Investigate the stability of this system. First, we find the steady state of the system from equations:

$$
\left\{\begin{array}{l}
p x_{0}-d_{0} x_{0}-\lambda_{0} x_{0} x_{2}=0 \\
2 d_{0} x_{0}-\lambda_{1} x_{1}=0 \\
s x_{1}-\lambda_{2} x_{2}-\lambda_{0} x_{0} x_{2}=0
\end{array}\right.
$$

There are two equilibrium positions, one of which is the trivial equilibrium that is unstable. Therefore, let consider a non-zero equilibrium position. It looks like this:

$$
\left(\frac{\lambda_{1} \lambda_{2}\left(p-d_{0}\right)}{\lambda_{0}\left(2 d_{0} s-\lambda_{1}\left(p-d_{0}\right)\right)} ; \frac{2 d_{0} \lambda_{2}\left(p-d_{0}\right)}{\lambda_{0}\left(2 d_{0} s-\lambda_{1}\left(p-d_{0}\right)\right)} ; \frac{\left(p-d_{0}\right)}{\lambda_{0}}\right) .
$$

To examine the stability of the steady state consider the matrix of the linearization of (2) at the steady state. It has the form: 


$$
\left(\begin{array}{ccc}
0 & 0 & -\frac{\lambda_{1} \lambda_{2}\left(p-d_{0}\right)}{2 d_{0} s-\lambda_{1}\left(p-d_{0}\right)} \\
2 d_{0} & -\lambda_{1} & 0 \\
-p+d_{0} & s & -\frac{2 d_{0} s \lambda_{2}}{2 d_{0} s-\lambda_{1}\left(p-d_{0}\right)}
\end{array}\right)
$$

Compose the characteristic equation of this matrix. For this let calculate the determinant and equate it to zero:

$\left|\begin{array}{ccc}-\mu & 0 & -\frac{\lambda_{1} \lambda_{2}\left(p-d_{0}\right)}{2 d_{0} s-\lambda_{1}\left(p-d_{0}\right)} \\ 2 d_{0} & -\lambda_{1}-\mu & 0 \\ -p+d_{0} & s & -\frac{2 d_{0} s \lambda_{2}}{2 d_{0} s-\lambda_{1}\left(p-d_{0}\right)}-\mu\end{array}\right|=0$,

Or

$\mu^{3}+\alpha \mu^{2}+\beta \mu+\gamma=0$,

Where

$\alpha=\frac{2 d_{0} s \lambda_{2}+2 d_{0} s \lambda_{1}-\lambda_{1}^{2}\left(p-d_{0}\right)}{2 d_{0} s-\lambda_{1}\left(p-d_{0}\right)}$,

$\beta=\frac{2 d_{0} s \lambda_{1} \lambda_{2}-\lambda_{1} \lambda_{2}\left(p-d_{0}\right)^{2}}{2 d_{0} s-\lambda_{1}\left(p-d_{0}\right)}$,

$\gamma=\frac{2 d_{0} s \lambda_{1} \lambda_{2}\left(p-d_{0}\right)-\lambda_{1}^{2} \lambda_{2}\left(p-d_{0}\right)^{2}}{2 d_{0} s-\lambda_{1}\left(p-d_{0}\right)}$.

The Hurwitz's matrix for the characteristic equation is

$\left(\begin{array}{lll}\alpha & 1 & 0 \\ \gamma & \beta & \alpha \\ 0 & 0 & \gamma\end{array}\right)$

According to the criterion of Routh-Hurwitz, the characteristic polynomial has roots with negative real part, and hence steady state of system (2) is stable, if

$\Delta_{1}=\alpha>0, \Delta_{2}=\alpha \beta-\gamma>0, \Delta_{3}=\gamma \Delta_{2}>0$. 
However, the characteristic equation has purely imaginary roots when $\Delta_{2}=0$.

Indeed, if $\lambda_{1,2}= \pm i \omega$ are the roots of equation (4), then, substituting in (4), we get:

$$
\begin{aligned}
& \lambda_{1}:-i \omega^{3}-\alpha \omega^{2}+i \beta \omega+\gamma=0, \\
& \lambda_{2}: i \omega^{3}-\alpha \omega^{2}-i \beta \omega+\gamma=0 .
\end{aligned}
$$

Adding these two equations, we get $\gamma=\alpha \omega^{2}$.

Subtract the second equation from the first. Then $\beta=\omega^{2}$, and, therefore, $\gamma=\alpha \beta$.

On the other hand, $\Delta_{2}=\alpha \beta-\gamma$. So, $\Delta_{2}=0$.

Consider the case, when the second order minor of the Hurwitz's matrix is equal to zero, and find such values of the parameter $s$, under which it is made.

$$
\begin{aligned}
& \Delta_{2}=\frac{2 d_{0} s \lambda_{2}+2 d_{0} s \lambda_{1}-\lambda_{1}^{2}\left(p-d_{0}\right)}{2 d_{0} s-\lambda_{1}\left(p-d_{0}\right)} \cdot \frac{2 d_{0} s \lambda_{1} \lambda_{2}-\lambda_{1} \lambda_{2}\left(p-d_{0}\right)^{2}}{2 d_{0} s-\lambda_{1}\left(p-d_{0}\right)}- \\
& -\frac{2 d_{0} s \lambda_{1} \lambda_{2}\left(p-d_{0}\right)-\lambda_{1}^{2} \lambda_{2}\left(p-d_{0}\right)^{2}}{2 d_{0} s-\lambda_{1}\left(p-d_{0}\right)}=0 ; \\
& s=\frac{\left(\lambda_{2}-\lambda_{1}\right)\left(p-d_{0}\right)^{2}+\lambda_{1}^{2}\left(p-d_{0}\right)}{2 d_{0}\left(\lambda_{1}+\lambda_{2}-\left(p-d_{0}\right)\right)} .
\end{aligned}
$$

Hence, at this value of the parameter $s$ the characteristic equation (4) has purely imaginary roots. It is easy to verify that it will satisfy to all conditions of the AndronovHopf bifurcation theorem. This means that for $s>s_{0}$ there are stable solutions, at $s=$ $s_{0}$ the equilibrium position is the center, and if $s<s_{0}$ there are an unstable solution and a stable limit cycle.

\section{Example}

Let $p=0.6, d_{0}=0.4, \lambda_{0}=\frac{1}{4000}, \lambda_{1}=0.03, \lambda_{2}=1$.

The corresponding steady state is

$\left(\frac{24}{0.8 s-0.006} ; \frac{640}{0.8 s-0.006} ; 800\right)$.

And the critical value $s=s_{0}=0.0587$. 


$$
\begin{aligned}
& \mu^{3}+1.17647 \mu^{2}+0.0051 \mu+0.006=0 ; \\
& \mu_{1}=-1.17647, \mu_{2,3}= \pm 0.07141 i .
\end{aligned}
$$

A non-trivial equilibrium has the form: $(585.88235 ; 15623.52941 ; 800)$.

The trajectory is presented in the following figure:

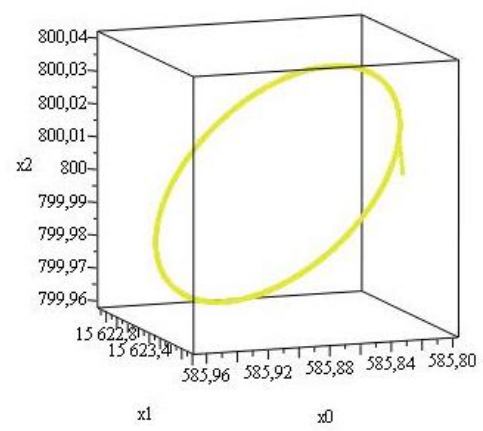

Fig. 1. The trajectory for $\mathrm{s}=0.0587$

Now consider the case when $s<s_{0}, s=0.05$.

The steady state has the form:

(705.88235; 18823.529; 800).

The trajectory is presented in the following figure:

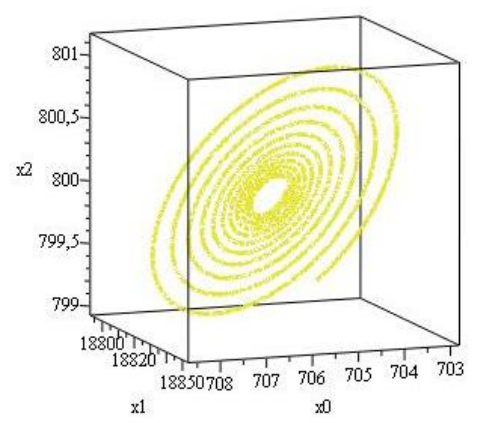

Fig. 2. The trajectory for $\mathrm{s}=0.05$.

In this case the steady state is unstable. Now consider the case when $s>s_{0}, s=0.1$.

The steady state has the form:

$(324.32432 ; 8648.64865 ; 800)$.

The trajectory is presented in the following figure: 


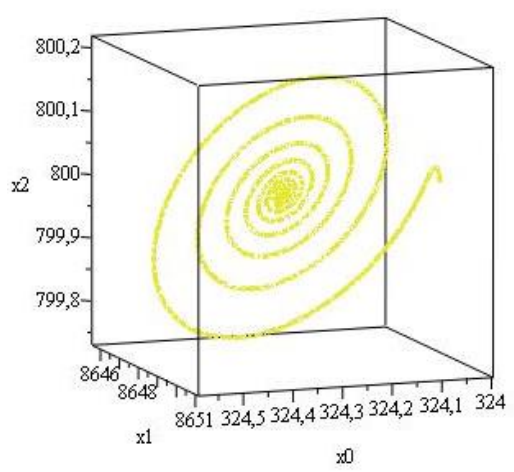

Fig. 3. The trajectory for $\mathrm{s}=0.1$.

In this case the steady state is asymptotically stable.

\section{Conclusion}

It is shown in the paper that for $n=1$ the Andronov-Hopf bifurcation takes place in the dynamical model under consideration. The phenomenon of "soft loss of stability" was stated in this model, see also [3-5].

\section{Acknowledgements}

This work is supported in part by the Russian Foundation for Basic Research (grant 14-01-97018-p) and the Ministry of Education and Science of the Russian Federation under the Competitiveness Enhancement Program of Samara University (2013-2020)

\section{References}

1. Marsden JE, McCracken M. The Hopf bifurcation and its applications. N.Y.: SpringerVerlag, 1976

2. Sanchez-Taltavull D, Alarcon T. Robustness of differentiation cascades with symmetric stem cell division. J. R. Soc. Interface, 2014; 11: 20140264. DOI: 10.1098/rsif.2014.0264.

3. Shchepakina E. Canards and black swans in model of a 3-D autocatalator. Journal of Physics: Conference Series, 2005; 22: 194-207.

4. Shchepakina E, Korotkova, O. Condition for canard explosion in a semiconductor optical amplifier. Journal of the Optical Society of America B: Optical Physics, 2011; 28(8): 1988-1993.

5. Shchepakina E, Korotkova O. Canard explosion in chemical and optical systems. Discrete and Continuous Dynamical Systems - Series B, 2013; 18(2): 495-512. 